\title{
Preparation of Fiber Reinforced Silica Aerogel Block
}

\author{
Shuning Zhai ${ }^{1}$, Chi Cao ${ }^{1}$, Yang Liu ${ }^{1}$, Chen Gui ${ }^{1}$, Kai Yuan ${ }^{1}$, Qun Gao ${ }^{\text {I, }}$, Benjamin Wilson ${ }^{2}$ \\ ${ }^{1}$ School of Shanghai Institute of Technology University, Shanghai, China \\ ${ }^{2}$ School of Nanyang Technological University, Singapore
}

\begin{abstract}
Silica aerogels are lightweight, highly porous nanomaterial with extremely large internal surface area but fragile and brittleness. To enhance the applicability of aerogels, fiber reinforced silica aerogel insulation blocks are prepared by frothing method. Polyurethane emulsion acts as binder whereas polyethylene (PE) fiber and polyurethane (PU) fiber is used as reinforcing phase. The effect of polyethylene fiber and polyurethane fiber on the performance of composites were characterized by material's chemical microscopic morphology, compression performance, recovery performance, surface area, thermal conductivity and hydrophobicity. The research results show that the prepared PU fiber reinforced silica aerogel insulation block has excellent comprehensive performance. The water absorption rate of decreased from $19.35 \%$ ( $0 \mathrm{phr}$, PU fiber) to $6 \%$ (20 phr, PU fiber), and comprehensive rebound rate was increased by $97.83 \%$ at $20 \mathrm{phr}$ PU fiber compared with pure insulation block (83.88\%). Meanwhile, the dimensional stability of the composite aerogel block is above $96 \%$. PU fiber reinforced silica aerogel insulation block has light weight, good hydrophobicity and good thermal and mechanical properties, so it has a wide range of application prospects in the field of insulation.
\end{abstract}

\section{Introduction}

Aerogel has porous structure formed by interconnecting three-dimensional nano network frameworks, so it has high porosity $(80 \% \sim 99.8 \%)[1]$, ultra-high specific surface area $(800 \sim 1000 \mathrm{~m} 2 / \mathrm{g})[2]$, super low thermal conductivity $[0.001 \sim 0.043 \mathrm{~W} /(\mathrm{m} \cdot \mathrm{K})][3]$ and very low density $(0.003 \sim 0.5 \mathrm{~g} / \mathrm{cm} 3)[4]$. And it is one of the key materials in the field of high-performance thermal insulation at present. However, pure silica aerogel has low mechanical properties and is brittle, which limits its industrial application. Therefore, it is necessary to strengthen the silica aerogel to prepare the silica aerogel thermal insulation composite material with strong mechanical properties[4].

Kim et al. prepared a glass fiber/silica aerogel composite insulation material and found that glass fiber can effectively enhance the toughness of Silica aerogel[5]. Zhang et al. explored the use of aluminum silicate fiber and glass fiber as silica aerogel reinforcements, and found that the composite filled with fibers and silica aerogel increased in compression strength than the fiber mat alone after $30 \%$ compression deformation. The composite material composed of glass fiber and Silica aerogel has more than $200 \%$ higher mechanical strength than aluminum silicate fiber[6]. Li et al. compounded sepiolite fibers with silica aerogel, which has good interfacial interaction to improve the mechanical properties of aerogel materials [7]. Zhou et al. added polyester fiber to silica aerogel, and the pressure carried by the material increased by $40 \mathrm{kPa}$ compared with pure silica aerogel when the elastic deformation became $80 \%[8]$. However, silica aerogels are generally prepared into powders through the sol-gel method, solvent replacement and supercritical drying processes, and it is difficult to make blocks and directly use them as thermal insulation materials. Generally, silica aerogel is compounded on the fiber mat to prepare a block with strong mechanical properties, or the aerogel is first made into powder, and then placed between two plates to form a sandwich-like insulation block, the thermal insulation performance of this sandwich product is difficult to reach the ideal level of super thermal insulation materials.

Water-based polyurethane (WPU) not only has the excellent properties of polyurethane (flexibility, adhesion, etc.), but also reduces the amount of organic solvents (or even to zero). It is a new type of environmentally friendly material, so it has received many widespread concern. Polyurethane binders are based on the unique chemical properties of isocyanates. Isocyanate is a compound containing an isocyanate group (-NCO) in the molecule. This group has a highly unsaturated bond structure with overlapping double bonds and can react with various compounds containing active hydrogen.

In this paper, combining silica aerogel with low thermal conductivity and water-based polyurethane emulsion with high cohesiveness, the two are combined by a wet process to prepare silica aerogel insulation block; further with $\mathrm{PE}$ Fiber and PU fiber are used as reinforcement, and a fiberreinforced silica aerogel insulation block with a size of $150 \mathrm{~mm} \times 150 \mathrm{~mm} \times 10 \mathrm{~mm}$ is prepared. Also, we compare and study the influence of different fibers content on the structure and performance of materials, in order to obtain thermal insulation blocks with excellent

\footnotetext{
$\overline{\text { * Corresponding author: wityy@sit.edu.cn }}$
} 
thermal insulation performance, so that large-scale and environmentally-friendly production of thermal insulation materials becomes possible.

\section{Experiment}

\subsection{Main materials}

The raw materials used in this experiment mainly include water-based polyurethane emulsion (Zhongke Huayu Technology Development Company Limited.), sodium dodecyl sulfonate (Titan Technology Company Limited.), Silica aerogel (Shenzhen Zhongning Technology Company Limited.), polyethylene fiber, polypropylene fiber and distilled water.

\subsection{Material preparation}

Weigh $20 \mathrm{phr}$ of water-based polyurethane emulsion, 2 phr of sodium dodecyl sulfonate, polyethylene fiber and polyurethane fiber and add $0 \mathrm{phr}, 5 \mathrm{phr}, 10 \mathrm{phr}, 15 \mathrm{phr}$, and $20 \mathrm{phr}$, respectively. Put the above raw materials into a high-speed mixer and stir for 30 minutes, then add 15 phr of Silica aerogel, and stir again for 30 minutes. The mixed homogeneous slurry is obtained, poured into a mold, and placed in a thermostatic oven for 48 hours at a constant temperature of $50^{\circ} \mathrm{C}$. to obtain a fiber-reinforced silica aerogel insulation block.

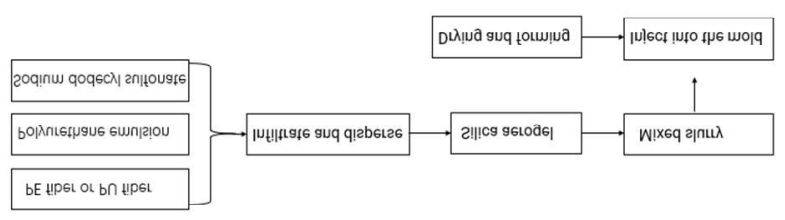

Figure 1. Preparation process

\subsection{Testing and characterization}

Field emission scanning electron microscope (FESEM) was performed on a S-3400N instrument to observe the microscopic appearance of the sample; the thermal conductivity meter was determined on a DRPL-III instrument to analyze the thermal conductivity of the sample; the water absorption per unit volume of the sample was calculated by water immersion method: 1) measure the mass before water (m1) and after water (m2); 2) water absorption $\mathrm{w}=[(\mathrm{m} 2-\mathrm{m} 1) / \mathrm{m} 1]^{*} 100 \%$; the compressive strength of the sample was measured on a Sun 500 universal material testing machine; the compression resilience rate of the sample was performed on a Sun 500 universal material testing machine: 1) measure the initial thickness $\mathrm{h} 0$ and recovery thickness $\mathrm{h} 1$; 2) Compression resilience rate $\mathrm{R}=(\mathrm{h} 1 / \mathrm{h} 0) * 100 \%$; the dimensional stability of the sample was determined by the constant temperature drying method: 1) Use a vernier caliper to measure the size before drying $(\mathrm{a} 0)$ and the size after drying (a1); 2) Dimensional stability $\mathrm{D}=$ (a1/a0)*100\%; Brunner-Emmet-Teller (BET) was performed on a ASAP2460 instrument to observe the surface area of the sample

\section{Results and discussion}

\subsection{The irfiuence of fiber types on the microscopic morphology of silica aerogel insulation blocks}

The microscopic morphology of the composite material can intuitively describe the composite situation of fiber and silica aerogel. Figure 2(a) is the SEM image of silica aerogel insulation block prepared without fiber. It can be seen from the figure that a large amount of Silica aerogel is combined through PU emulsion to form needle-shaped particles to form a network structure, and there are a lot of pores inside. It can be seen from figure 2(b) and (c) that PE fiber and PU fiber are embedded in silica aerogel insulation block, while silica aerogel is attached to the surface of two kinds of fibers, which proves that silica aerogel and fiber form a better interface bonding. The interfacial combination provides strong evidence for the improvement of mechanical properties of composite materials. The gap between the fiber and the fiber is large, which reduces the thermal bridge effect during contact and reduces the thermal conductivity of the composite material.

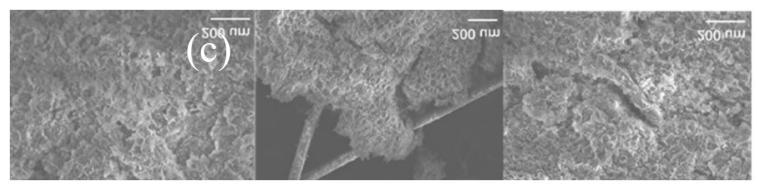

Figure 2. SEM images of silica aerogel insulation blocks: (a) No fiber added; (b) PE fiber (5 phr); (c) PU fiber (5 phr).

\subsection{The influence of fiber type on the thermal conductivity of silica aerogel insulation blocks}

Thermal conductivity refers to the amount of heat transferred through a unit horizontal cross-sectional area in a unit time when the temperature vertical downward gradient is $1{ }^{\circ} \mathrm{C} / \mathrm{m}$. Figure 3 shows the thermal conductivity of the silica aerogel insulation block with different fiber additions. With the increase of PE fiber addition, the thermal conductivity of the composite material gradually increased, reaching a maximum of $0.02953 \mathrm{~W} /(\mathrm{m} \cdot \mathrm{K})$; however, with the increase of PU fiber, the thermal conductivity of silica aerogel insulation block increases first, then decreases and then increases, reaching a maximum of $0.02387 \mathrm{~W} /(\mathrm{m} \cdot \mathrm{K})$. The fiber can shorten the solid phase transmission path, reduce the heat resistance, increase the solid phase heat conduction, and increase the thermal conductivity. Therefore, the thermal conductivity of the aerogel insulation block with the fiber is greater than that when it is not added. 


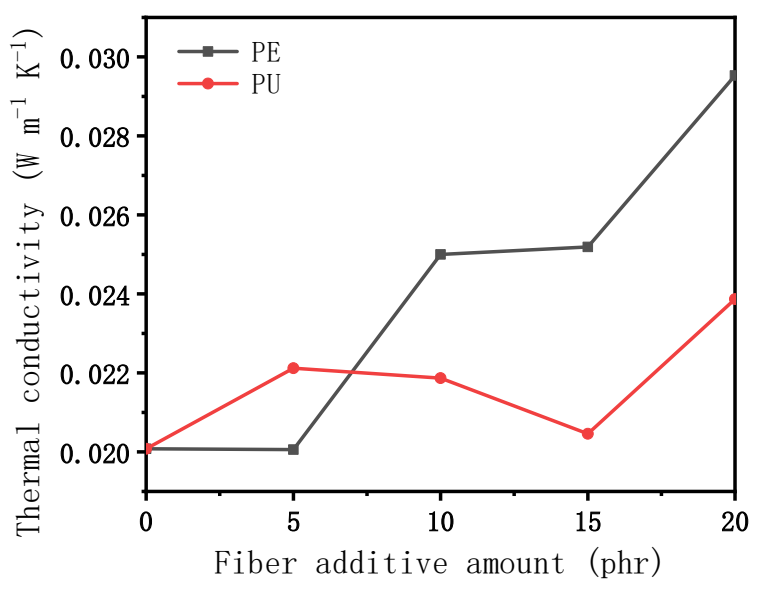

Figure 3. Thermal conductivity image of silica aerogel insulation blocks.

\subsection{The influence of fiber type on the water absorption of silica aerogel insulation blocks}

Water absorption is a physical quantity that indicates the degree of water absorption by an object under ambient pressure. Figure 4 is a graph of water absorption of silica aerogel insulation blocks with different fiber additions. Polyurethane has weak water absorption as binding agent, and the two fibers have better moisture resistance, the water absorption rate of the silica aerogel insulation block decreases with the fiber content increases. When the amount of PE fiber added is $20 \mathrm{phr}$, the composite material has a loose structure and more pores, so a large amount of water can be stored inside, resulting in an increase in the water absorption rate of the material. When the amount of PU fiber added is $20 \mathrm{phr}$, the water absorption of the silica aerogel insulation block reaches the lowest value of $6 \%$.

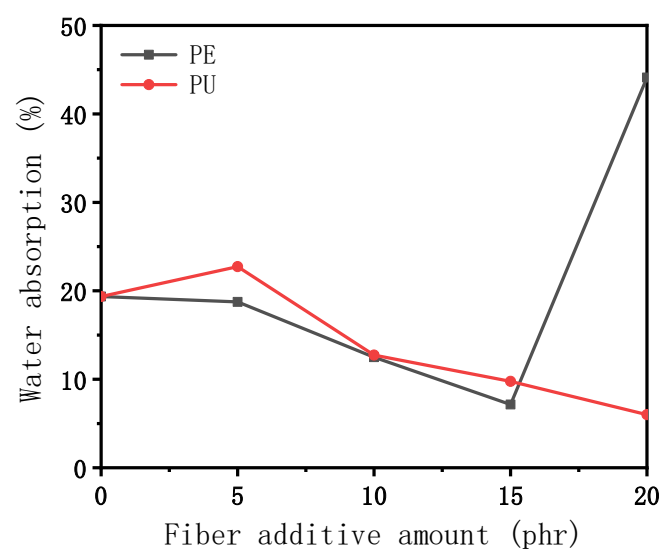

Figure 4. Water absorption image of silica aerogel insulation blocks.

\subsection{The influence of fiber types on the compressive strength of silica aerogel insulation blocks}

Compressive strength is the compressive stress of the sample when it is deformed by $20 \%$. It characterizes the material's ability to resist compressive loads without failure. Figure 5 is a graph of the compressive strength of silica aerogel insulation blocks with different fiber additions. For PE fiber, the contact area and interface binding force between aerogel and fiber are small, so as the external stress increases, the compressive strength decreases. For PU fiber, when the fiber content is small, the binding force of silica aerogel and fiber is stronger, and its compressive strength increases. When the amount of PU fiber added is $10 \mathrm{phr}$, the compressive strength of the composite material reaches the maximum value of $0.0323 \mathrm{MPa}$; when the fiber content is high, the interface between the silica aerogel and the fiber is weakly bonded, and its compressive strength is low. When the PU fiber addition amount is $20 \mathrm{phr}$, the composite material's compressive strength reaches the minimum value of $0.00575 \mathrm{MPa}$.

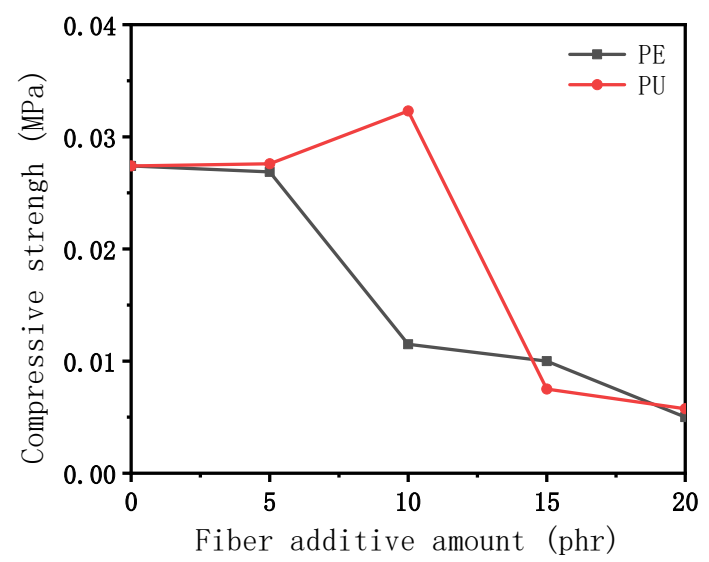

Figure 5. Compressive strength image of silica aerogel insulation blocks.

\subsection{The influence of fiber type on the compression rebound of silica aerogel insulation blocks}

Compression rebound rate is the degree to which the sample returns to its original state after being deformed by the applied force. Figure 6 shows the compression rebound of the silica aerogel insulation block with different fiber additions. As the amount of fiber increases, the compression rebound of the sample is improved. This is because the silica aerogel is supported by fibers. As the fiber content increases, the static air layer increases. From the side, it shows that the addition of fibers helps to improve the compression rebound of the silica aerogel insulation block. When the amount of PU fiber added is $20 \mathrm{phr}$, the compression rebound rate of the composite material reaches the maximum value of $97.83 \%$. 


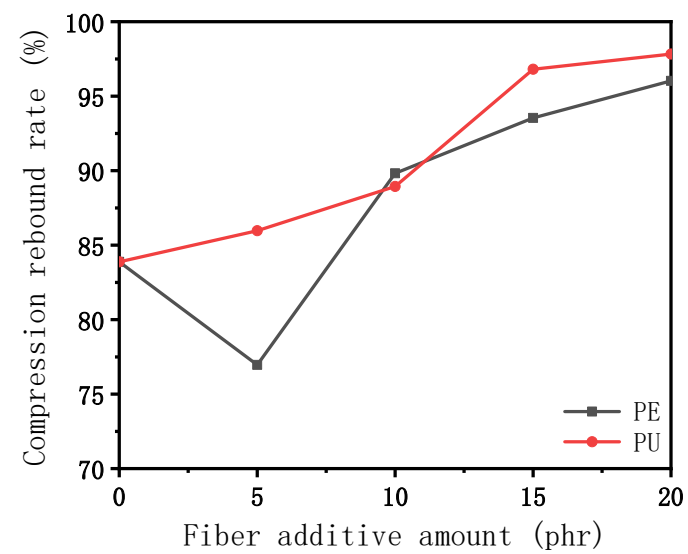

Figure 6. Compression rebound image of silica aerogel insulation blocks.

\subsection{The influence of fiber types on the dimensional stability of silica aerogel insulation blocks}

Dimensional stability refers to the performance of a material that does not change its external dimensions when it is affected by external conditions, and is an index to determine whether the material is prone to deformation. Figure 7 is a graph showing the dimensional stability of silica aerogel insulation blocks with different fiber additions. It can be found from the figure that the dimensional stability of the two different fibers is above $96 \%$, which proves that the material has good dimensional stability.

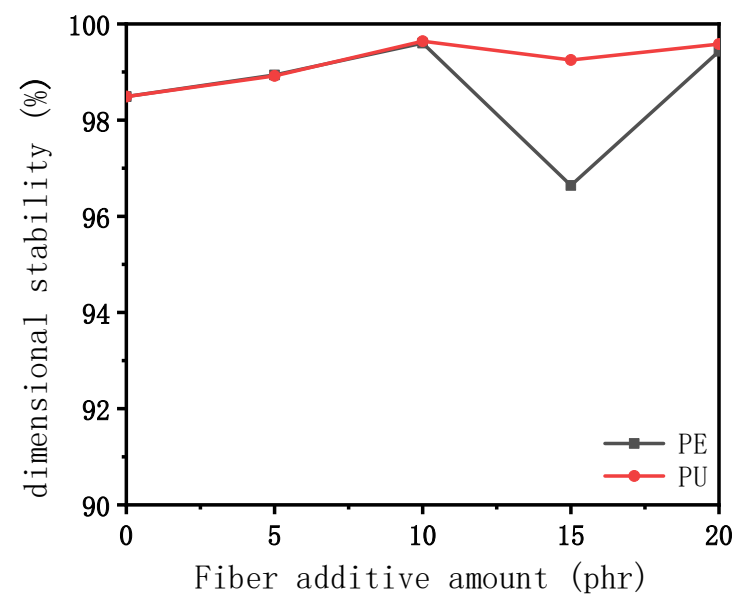

Figure 7. Dimensional stability image of silica aerogel insulation blocks.

\subsection{The influence of fiber types on the surface area of silica aerogel insulation blocks}

The surface area refers to the total area of a unit mass material. Figure 8 is a graph showing the surface area performance of silica aerogel insulation blocks with different fiber additions. It can be found from the figure that the specific surface area of the aerogel insulation block is the largest $(288.32 \mathrm{~m} 2 \mathrm{~g}-1)$ when no fiber is added. With the increase of the content of the two fibers, the surface area of the aerogel insulation block is decreasing. The test results show that the addition of fibers has a certain effect on the aerogel insulation block, increasing the pores of the aerogel insulation block and reducing its surface area.

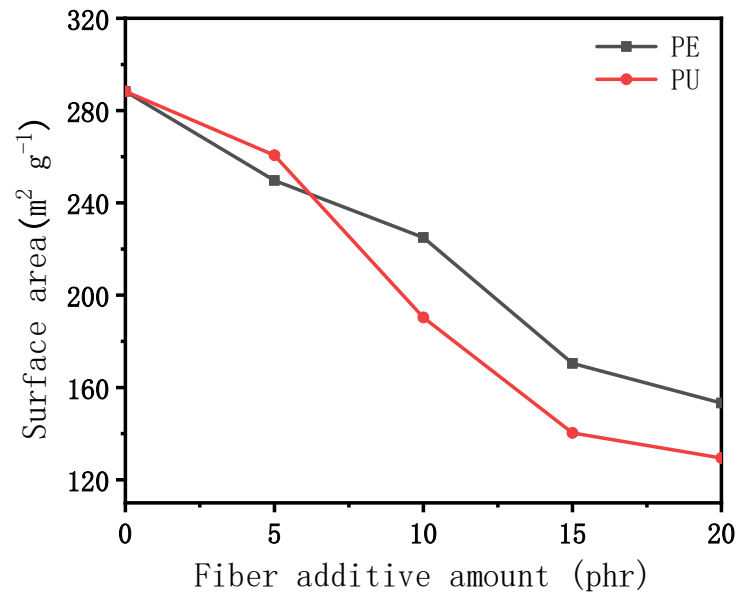

Figure 8. Surface area image of silica aerogel insulation blocks.

\section{Conclusion}

Using silica aerogel as the powder, polyethylene fiber and polyurethane fiber as the reinforcing phase, and polyurethane emulsion as the binding agent, the silica aerogel insulation blocks with better thermal insulation performance are prepared by the frothing method and ambient drying method.

Explore the effects of polyethylene fiber and polyurethane fiber and their added content on the thermal conductivity, water absorption, compression rebound, surface area, compression strength and dimensional stability of silica aerogel insulation blocks. The results show that the overall performance of the polyurethane fiber reinforced silica aerogel insulation block with $10 \mathrm{phr}$ is the best. The thermal conductivity is $0.02187 \mathrm{~W} /(\mathrm{m} \cdot \mathrm{K})$, the water absorption rate is $12.73 \%$, the surface area is 190.34 $\mathrm{m}^{2} \mathrm{~g}^{-1}$, the compression rebound rate is $81.77 \%$, and the compression strength (20\% Strain) is $0.0323 \mathrm{Mpa}$.

\section{Acknowledgments}

This work was financially supported by postgraduate high-level applied scientific research results fund.

\section{References}

1. H. Suo, W. Wang, S.J. Jiang, et al, Research progress of high temperature resistant aerogel insulation materials, Shanghai Aerospace. 36 (2019) 61-68.

2. X.Q. Jiang, Research progress of silica aerogels, Fine and Specialty Chemicals. 28 (2020) 42-46.

3. Y.Z. Lu, R. Chen, Y.Z. Li, et al, Hydrophobic modification of silica aerogels and research progress 
of composite materials, Shandong Chemical Industry. 49 (2020) 72-73.

4. L. Xu, Y.G. Jiang, J.Z. Feng, et al, Preparation of fiber-reinforced A12O3- $\mathrm{SiO} 2$ aerogel thermal insulation composite material and thermal insulation performance, Journal of Nanjing University of Technology (Natural Science Edition). 42 (2020) 461-466.

5. S. Kim, J. Seo, J. Cha, et al, Chemical retreating for gel-typed aerogel and insulation performance of cement containing aerogel, Construction And Building Materials. 40 (2013) 501-505.

6. Y. Zhang, X.G. Ge, G.J. Lu, et al, Preparation and properties of aluminum silicate fiber and glass fiber composite silica aerogel materials, Inorganic Salt Industry. (2020): 1-5.

7. X. Li, Q. Wang, H. Li, et al, Effect of sepiolite fiber on the structure and properties of the sepiolite/silica aerogel composite, Journal of sol-fel science and technology. 67 (2013) 646- 653.

8. J.L. Zhou, M.J. Shi, D. Long, et al, Preparation and properties of silica aerogel toughened by polyester fiber, Functional Materials. 49 (2018) 10150-10154. 